(с) И.В. Кононенко, А.А. Глибка*, Н.А. Зубкова, А.Ю. Майоров, А.Н. Тюльпаков, О.М. Шмидт

Национальный медицинский исследовательский центр эндокринологии, Москва

Традиционно около 90\% всех случаев сахарного диабета (СД) у взрослых приходится на долю СД 2 типа, при этом, как правило, СД типа MODY (maturity-onset diabetes of the young - диабет взрослого типа у молодых лиц) остается вовремя не диагностированным, что, в свою очередь, приводит к выбору неправильной тактики лечения. Одной из наиболее распространенных моногенных форм СД является заболевание, обусловленное мутацией гена глюкокиназы (GCK) - СД типа MODY2. Знание особенностей клинической картины заболевания позволяет выделить пациентов с высоким риском наличия мутации гена GCK и направить их для верификации диагноза на молекулярно-генетическое исследование (МГИ).

В настоящей работе отражены особенности клинической картины СД типа MODY2 и трудности его диагностики у взрослых. Представлен клинический случай пациентки с СД типа MODY2, который в полной мере демонстрирует все особенности данного типа СД.

КЛЮЧЕВЫЕ СЛОВА: сахарный диабет; ген глюкокиназы (GCK); диабет взрослого типа у молодых лиц (MODY); CД типа MODY2; гестационный сахарный диабет; клинический случай

\title{
MODY2 DIAGNOSTIC ISSUES IN ADULTS
}

(c) Irina V. Kononenko, Anastasia A. Glibka*, Alexander Y. Mayorov, Natalya A. Zubkova, Anatoly N. Tyulpakov, Olga M. Schmidt

Endocrinology Research Centre, Moscow, Russia

Approximately $90 \%$ of all cases of diabetes mellitus in adults involve type 2 diabetes, while the prevalence of maturityonset diabetes of the young (MODY) remains undetermined leading to inappropriate treatment regimens. One of the most common monogenic forms of diabetes is a disease caused by a mutation in the glucokinase gene, MODY2. Knowledge of the clinical features of the disease allows the selection of patients with a high risk of mutation in the glucokinase gene and verification of diagnosis for molecular genetic research. This paper reflects the clinical features of MODY2 and the difficulties of diagnosis in adults. Furthermore, it presents a clinical case of a patient with MODY2 demonstrating all the features of this type of diabetes. A family member with a mutation in the gene allows to predict the nature of carbohydrate metabolism disorders in first degree relatives. A targeted study of only one part of the glucokinase gene in molecular genetic research is sufficient to confirm the diagnosis in relatives.

KEYWORDS: diabetes mellitus; GCK gene; maturity-onset diabetes of the young (MODY); MODY2; gestational diabetes; case report

В типичных случаях для определения типа сахарного диабета (СД) и выбора дальнейшей тактики лечения достаточно анализа клинической картины заболевания. Дополнительные иммунологические и генетические исследования, позволяющие провести дифференциальную диагностику различных типов СД, проводятся редко. Вместе с тем ряд исследований показывает, что от 7 до 15\% случаев СД неправильно классифицированы [1]. С расширением возможностей и доступности молекулярно-генетических исследований (МГИ) становится очевидным, что распространенность моногенных форм СД выше, чем было принято считать ранее. В ранее проведенных исследованиях было показано, что в детском и подростковом возрасте частота моногенных форм СД составляет 1,1-4,25\% всех форм СД, а распространенность в популяции - 2,1-4,6 на 100000 [2]. Среди американцев моложе 20 лет около 10\% случаев СД при наличии остаточной секреции инсулина и отсутствии антител к различным антигенам поджелудочной железы на самом деле являются случаями моногенного СД [3]. По данным других авторов, частота моногенных форм СД в развитых странах составляет 1-2\% [4]. При этом в 2010 г. S. Amed и соавт. [5] сообщили о минимальном уровне заболеваемости MODY в Канаде среди исследуемых в возрасте до 18 лет: 0,4 случая на 100 000. В 2012 г. T. Della Manna и соавт. [6] отметили, что распространенность в Бразилии MODY варьировала от 0,3\% до 2,4\% случаев диабета, из них примерно 70\% случаев приходится на MODY3 и MODY2. Относительная распространенность подтипов MODY различается. В Чехии S. Pruhova и соавт. [7] продемонстрировали многолетнее исследование (с 1999 по 2009 гг.), где было проведено МГИ у 959 пробандов из 292 семей, демонстрирующее частоту выявляемости моногенных форм СД: 35\% для MODY2, 10,6\% для MODY3, 4,5\% для MODY1. В исследовании 59 израильских пациентов с MODY в 2007 г. [8] была выявлена относительная 
частота MODY: 10,1\% для MODY3, 8,5\% для MODY2, 1,7\% для MODY1.

MODY объединяет формы СД с аутосомно-доминантным типом наследования, в основе которых лежат мутации генов, ассоциированных с нарушением функции бета-клеток.

Более $80 \%$ случаев MODY остаются недиагностированными, и часто требуется более 10 лет, прежде чем удается верифицировать диагноз [9].

В настоящее время известно 14 генов, приводящих к развитию различных типов MODY [10], при этом наиболее часто встречаются две формы - MODY2 и MODY3, обусловленные мутациями в генах глюкокиназы (GCK) и ядерного фактора гепатоцитов (HNF1a) соответственно $[2,10]$.

При MODY2 клинические симптомы заболевания обычно отсутствуют, выявление нарушений углеводного обмена часто носит случайный характер (при проведении профилактического обследования или по поводу сопутствующего заболевания, а также при скрининге во время беременности). Пациентам не требуется назначение инсулинотерапии - нормогликемию удается поддерживать с помощью диеты. Исключение составляют беременные с СД типа MODY2, когда инсулинотерапия предотвращает развитие макросомии плода в случае отсутствия у него аналогичной мутации [11-14].

Гомозиготная инактивирующая мутация в GCK может приводить к полному дефициту глюкокиназы и быть причиной перманентного неонатального СД [12]. Если же в гене GCK имеются активирующие мутации, развивается уникальная форма врожденного гиперинсулинизма [15].

Знание основных клинических особенностей СД типа MODY2 позволяет на этапе сбора анамнеза предположить наличие данной формы СД, провести необходимое обследование и направить пациента на МГИ. Обнаружение мутации в гене GCK позволяет однозначно верифицировать диагноз СД типа MODY2.

\section{ОПИСАНИЕ КЛИНИЧЕСКИХ СЛУЧАЕВ}

Применение методов МГИ в отделении терапии диабета Института диабета ФГБУ «НМИЦ эндокринологии» Минздрава России в течение 2017-2018 гг. позволило диагностировать 12 случаев СД типа MODY2.

Молекулярно-генетический анализ проводился в лаборатории отделения наследственных эндокринопатий ФГБУ «НМИЦ эндокринологии» МЗ РФ (зав. отд. д.м.н. Тюльпаков А.Н.). Геномную ДНК выделяли из лейкоцитов периферический крови стандартным методом (набор Pure Link, Genomic DNA Mini Kit, Life Technologies, США). Для молекулярно-генетического анализа применялся метод NGS. Использовалась разработанная в отделении наследственных эндокринопатий ФГБУ «НМИЦ эндокринологии» МЗ РФ панель праймеров для мультиплексной ПЦР и секвенирования с применением технологии Ion Ampliseq $^{\text {TM }}$ Custom DNA Panel (Life Technologies, CШA). Секвенирование осуществлялось на полупроводниковом секвенаторе PGM (Ion Torrent, Life Technologies, США). Биоинформатическая обработка результатов секвенирования проводилась с помощью программного модуля Torrent Suite 4.2.1 (lon Torrent, Life Technologies, США) и пакета программ Annovar (версия 2014Nov12).
В качестве референсных последовательностей кДНК генов-кандидатов использовались ссылки Genbank. Интерпретация результатов исследований и оценка патогенности нуклеотидных изменений проводились согласно международным рекомендациям. Все единичные нуклеотидные варианты с частотой минорного аллеля более чем 0,001 были исключены из последующего анализа. Обозначение мутаций проводилось в соответствии с рекомендациями den Dunnen и Antonarakis. В последующем у родственников проводился поиск аналогичных мутаций на секвенаторе Genetic Analyzer Model 3130 (Life Technologies, США).

Медиана возраста пациентов на момент обследования составила 28 [22;34,25] лет (от 18 до 48 лет). При этом только у одного обследуемого генетическое исследование, подтверждающее диагноз, проведено незамедлительно, при первичном выявлении нарушений углеводного обмена. Нарушения углеводного обмена дебютировали в возрасте от 9 до 19 лет. Медиана длительности заболевания составила 10 [2;21] лет. Все пациенты были госпитализированы для уточнения типа СД, при этом у троих ранее выявленные нарушения углеводного обмена соответствовали критериям нарушенной толерантности к глюкозе (НТГ), двоим был поставлен диагноз СД 2 типа и одной - СД 1 типа. На момент проведения МГИ 6 пациентов придерживались диеты с ограничением легкоусвояемых углеводов, 2 пациентки получали инсулинотерапию, 3 - принимали вилдаглиптин (2 в комбинации с метформином) и 1 пациентка принимала метформин.

Медиана индекса массы тела (ИМТ) на момент обследования составила 20,65 [19,4;21,85] кг/м². Один пациент, у которого нарушения углеводного обмена были впервые диагностированы в возрасте 41 года, страдал

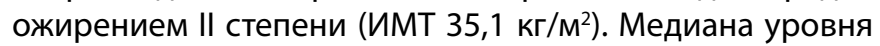
гликированного гемоглобина $\left(\mathrm{HbA}_{1 c}\right)$ обследуемых составила 6,4 [5,85;6,6] \%). Только у 4 из 13 уровень $\mathrm{HbA}_{1 с}$ на момент обследования соответствовал критериям СД $(\geq 6,5 \%)-6,6-6,7 \%$. У 3 пациентов уровень $\mathrm{HbA}_{1 с}$ при длительности заболевания более 20 лет составил 6,4\%, 6,3\%, $6,0 \%$ соответственно. Пероральный глюкозотолерантный тест (ПГТТ) был проведен 7 пациентам (табл. 1). По результатам ПГТТ у 3 пациентов нарушения углеводного обмена были классифицированы как СД, у 4 - как НТГ.

Ни у кого из пациентов не было выявлено микрососудистых осложнений СД, что особенно важно, учитывая длительность нарушения углеводного обмена.

Основные клинические характеристики представленной группы больных с СД типа MODY2 представлены в табл. 2.

Далее представлен клинический случай пациентки с СД типа MODY2, который в полной мере демонстрирует особенности данного типа СД.

Таблица 1. Показатели перорального глюкозотолерантного теста у пациентов с сахарным диабетом типа MODY2 (n=7, Me [Q1;Q3])

\begin{tabular}{ccc}
\hline $\begin{array}{c}\text { ПГТТ, } \\
\text { мин }\end{array}$ & $\begin{array}{c}\text { Глюкоза плазмы, } \\
\text { ммоль/л }\end{array}$ & $\begin{array}{c}\text { С-пептид плазмы, } \\
\text { нг/мл }\end{array}$ \\
\hline 0 & $6,69[6,27 ; 6,92]$ & $2,12[1,44 ; 40,89]$ \\
120 & $10,99[9,69 ; 11,40]$ & $10,10[6,62 ; 12,42]$ \\
\hline
\end{tabular}


Таблица 2. Клиническая характеристика пациентов с сахарным диабетом типа MODY2 (Me [Q1; Q3])

\begin{tabular}{|c|c|}
\hline Показатель & Значения \\
\hline Количество больных (м/ж), n & $12(4 / 8)$ \\
\hline Возраст на момент обследования, лет & $28[22 ; 34,25]$ \\
\hline Возраст дебюта нарушений углеводного обмена, лет & $4[13 ; 19]$ \\
\hline ИМТ, кг/м² & $20,65[19,4 ; 21,85]$ \\
\hline $\begin{array}{l}\mathrm{HbA}_{1 c^{\prime}} \% \\
\mathrm{HbA}_{1 \mathrm{c}} \geq 6,5 \%, \mathrm{n}\end{array}$ & $\begin{array}{c}\text { 6,4 [5,85; 6,6] } \\
4 \text { пациента }\end{array}$ \\
\hline Глюкоза плазмы натощак, ммоль/л & $6,69[6,27 ; 6,925]$ \\
\hline $\begin{array}{l}\text { Диагноз при поступлении (поставлен ранее), n: } \\
\text { - СД } 1 \text { типа } \\
\text { - СД } 2 \text { типа } \\
\text { - СД неуточненный } \\
\text { - НТГ }\end{array}$ & $\begin{array}{l}1 \\
2 \\
6 \\
3\end{array}$ \\
\hline $\begin{array}{l}\text { Терапия до верификации диагноза, n: } \\
\text { - диета } \\
\text { - пероральные препараты (метформин + ингибиторы ДПП-4) } \\
\text { - инсулинотерапия }\end{array}$ & $\begin{array}{l}6 \\
4 \\
2\end{array}$ \\
\hline $\begin{array}{l}\text { Модификация терапии после МГИ: } \\
\text { - диета }\end{array}$ & 12 \\
\hline
\end{tabular}

Примечания: СД - сахарный диабет; ИМТ - индекс массы тела; НТГ - нарушение толерантности к глюкозе; МГИ - молекулярно-генетические исследования; ДПП-4 - дипептидилпептидаза-4

Пациентка И., 35 лет, поступила в отделение с жалобами на повышение глюкозы плазмы натощак до 7,3 ммоль/л.

Из анамнеза известно, что впервые нарушение углеводного обмена выявлено в возрасте 14 лет (на фоне нормальной массы тела) при диспансеризации. В течение 2 лет наблюдалась по месту жительства с диагнозом НТГ, соблюдала диету с ограничением легкоусвояемых углеводов. При самоконтроле периодически отмечалось повышение гликемии натощак. С 16 лет не обследовалась. Колебания массы тела в течение 19 лет наблюдения составляли не более 7 кг (минимально 56 кг, максимально 63 кг).

В 23 года - самопроизвольное прерывание беременности на сроке 10 недель. В 33 года во время второй беременности на 11-й неделе диагностирован гестационный СД. Была назначена интенсифицированная инсулинотерапия, на фоне которой достигнуты целевые показатели гликемии (перед родами Левемир 16 Ед на ночь, НовоРапид по 9-10 Ед перед основными приемами пищи: 0,65 Ед/кг/сут). Вес до беременности 62,5 кг, перед родами - 71 кг (общая прибавка веса 8,5 кг). Роды срочные (38-я неделя гестации), путем кесарева сечения, вес ребенка при рождении 2560 г. Через 2 мес после родов проведен ПГТТ: гликемия натощак 6,6 ммоль/л, через 2 ч - 11,0 ммоль/л; С-пептид натощак 262 пмоль/л (0,79 нг/мл), через 2 ч - 1478 пмоль/л, тиреотропный гормон (ТTГ) - 0,46 мMЕ/л. Рекомендована низкоуглеводная диета.

Динамика $\mathrm{HbA}_{1 с}$ после родов: через 3 мес - 6,4\%; через год $-5,6 \%$. В связи с повышением гликемии натощак до 6,4-6,5 ммоль/л эндокринологом был назначен метформин с постепенным увеличением дозы до 1700 мг/сут. В связи с отсутствием эффекта препарат самостоятельно отменила. Пациентка была госпитализирована с целью уточнения диагноза.
При осмотре вес 56,5 кг, рост 165 см, ИМТ 20,8 кг/м². При обследовании: $\mathrm{HbA}_{1 c}-6,0 \%$, инсулин натощак 7,92 мкЕд/мл (2,3-26,4), антитела к антигенам бета-клеток не выявлены. В ходе ПГТТ выявлена НТГ на фоне сохранной секреции инсулина (глюкоза плазмы: 6,46-10,679,93 ммоль/л, С-пептид: 1,68-7,39-8,24 нг/мл). Повышение гликемии в ходе теста (через 2 ч) составило менее 4,6 ммоль/л [16-19]. На фоне обычного режима питания показатели гликемии натощак варьировали от 6,3 до 7,3 ммоль/л, после еды - максимально до 8,7 ммоль/л. При обследовании признаков диабетической ретинопатии, нефропатии не выявлено.

Семейный анамнез (рис. 1):

отец пробанда (II.2): 65 лет, на протяжении 40 лет отмечается незначительное повышение гликемии до 7,0 ммоль/л, лечения не получает;

единокровная сестра (III.7): 9 лет 4 мес (рост 131 см; вес 27,5 кг), ИМТ 16,0 кг/м² (-0,27 SD), бессимптомная непрогрессирующая гипергликемия натощак до 6,5 ммоль/л регистрируется с 2,5 лет. При обследовании в возрасте 8 лет 7 мес: $\mathrm{HbA}_{1 с}-6,1 \%$, инсулин - 3,6 мкЕд/мл (2,6-24,9), С-пептид - 293 пмоль/л (370-1470), антитела к антигенам бета-клеток отрицательные;

двоюродная сестра (III.9): 35 лет, ИМТ 17,2 кг/м². Активно выявлено повышение гликемии натощак 7,0 ммоль/л. При проведении ПГТТ: гликемия натощак 6,0 ммоль/л, через 2 ч - 12,3 ммоль/л;

двоюродный племянник (IV.6): 10 лет 8 мес (рост 134 см, вес 27 кг), ИМТ 15,0 кг/M² (-1,03 SD). Повышение гликемии натощак до 6,3 ммоль/л выявлено впервые случайно в возрасте 9 лет 2 мес, $\mathrm{HbA}_{1 с}$ 6,2\%. Рекомендовано соблюдение диеты с ограничением легкоусвояемых углеводов. При обследовании в ФГБУ «НМИЦ эндокринологии» МЗ РФ в 10 лет 3 мес $\mathrm{HbA}_{1 с}$ 6,7\%. Специфических панкреатических антител не обнаружено. При проведении ПГТТ 
I

II

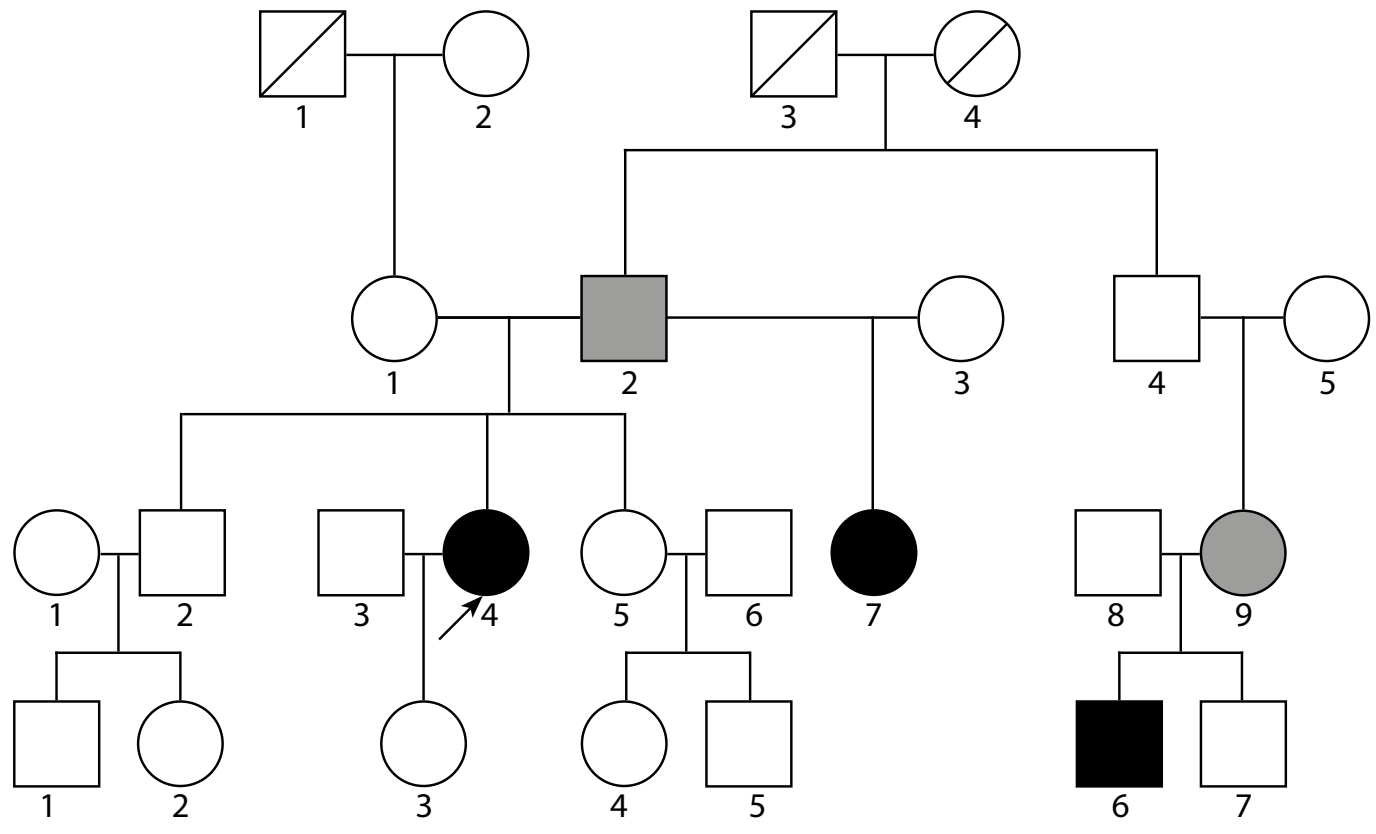

Обозначения:

римские цифры (I, II и др.) - поколение семьи

арабские цифры (1, 2 и др.) - представители одного поколения
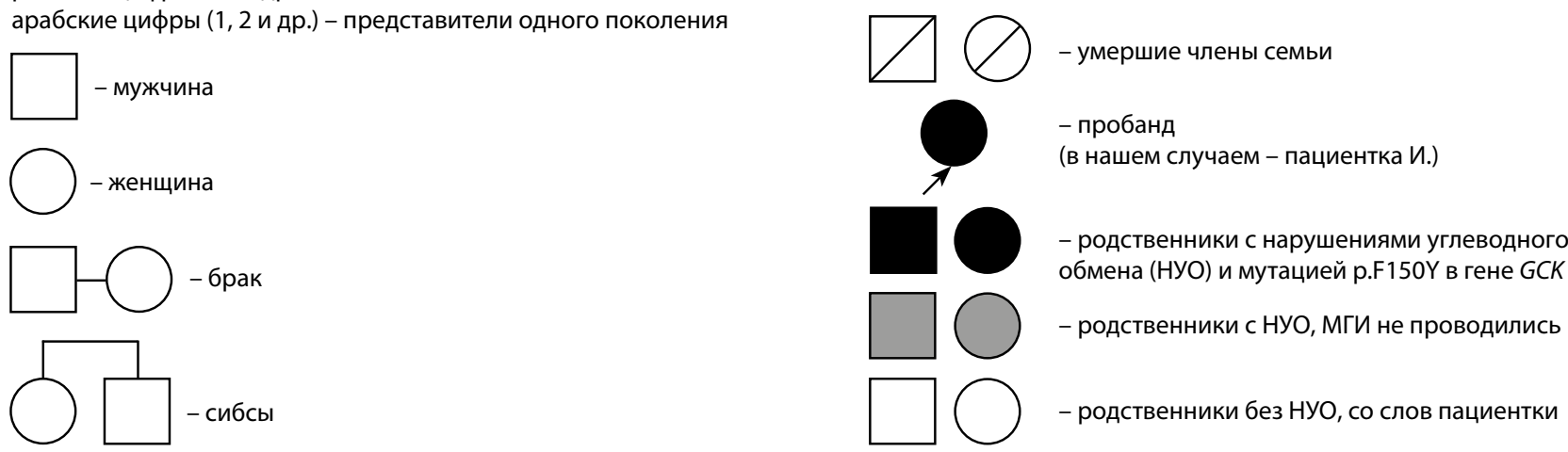

Рис. 1. Родословная семьи пациентки И. Примечания: НУО - нарушения углеводного обмена. МГИ - молекулярно-генетическое исследование

выявлена НТГ (глюкоза плазмы 6,12-8,0 ммоль/л) на фоне сохранной базальной и стимулированной секреции инсулина (инсулин 4,45-14,68 мкЕд/мл; С-пептид 1,0-2,86 нг/мл). Показатели гликемии натощак максимально - до 7,3 ммоль/л, после еды - до 10,9 ммоль/л.

Учитывая особенности течения заболевания, наследственный характер нарушения углеводного обмена, пациентке проведено МГИ. В гене GCК выявлен гетерозиготный вариант: с.449T>A:p.F150Y, описанный при СД типа MODY2 как патогенный. Аналогичные мутации выявлены у единокровной сестры и двоюродного племянника. Пациентке было рекомендовано нормокалорийное сбалансированное питание с ограничением легкоусвояемых углеводов, дозированные физические нагрузки, самоконтроль гликемии 1-2 раза в неделю в разное время суток.

\section{ОБСУЖДЕНИЕ}

Ген глюкокиназы (GCK) расположен на 7 хромосоме, имеет 10 кодирующих экзонов и кодирующую последовательность 1398 пар нуклеотидов. GCK - первый верифицированный ген-кандидат MODY (1992 г.) [20]. Основной функцией глюкокиназы является кодирование белка из семейства гексокиназ (IV изотип). Данный белок в основном экспрессируется в гепатоцитах и клетках поджелудочной железы и катализирует фосфорилирование глюкозы с образованием глюкозо-б-фосфата, что составляет первый этап в большинстве путей метаболизма глюкозы [21]. Активность данного фермента не ингибируется его продуктом - глюкозо-6-фосфатом, поэтому он сохраняет свою активность даже при избытке глюкозы. В бета-клетках поджелудочной железы глюкокиназа выполняет функцию датчика при изменении концентрации глюкозы в крови (своеобразный сенсор), и от ее ферментативной активности зависит скорость секреции инсулина, что и объясняет гипергликемию при инактивирующей мутации гена GCK (восприятие организмом гипергликемии как нормы). В печени глюкокиназа играет важную роль в поглощении глюкозы и превращении ее в гликоген. Определено, что мутации, обуславливающие развитие СД типа MODY2, непропорционально распределены в экзонах со со второй по десятую [22]. К 2018 г. описано 745 мутаций в гене GCK [23]. Частых мутаций в гене GCK не выявлено, каждая из них определена преимущественно в одной семье [2, 23, 24].

В нашей группе пациентов были выявлены следующие мутации гена глюкокиназы (табл. 3).

Большинство случаев СД в возрастной категории до 40 лет при нормальной массе тела классифицируются как СД 1 типа, реже - как СД 2 типа и редко подвергаются пересмотру. Отсутствие аутоантител как маркера СД 1 типа является крайне важной составляющей в дифференциальной диагностике моногенных форм СД у молодых 
Таблица 3. Спектр нуклеотидных изменений, выявленных в гене GCK

\begin{tabular}{|c|c|c|c|c|c|c|c|}
\hline Пациент & $\begin{array}{c}\text { Наследствен- } \\
\text { ность }\end{array}$ & $\begin{array}{c}\text { Нуклеотидная } \\
\text { замена }\end{array}$ & $\begin{array}{c}\text { Аминокислотная } \\
\text { замена }\end{array}$ & Экзон & gnomAD & HGMD & Патогенность \\
\hline N1 & 0 & c. $68 \mathrm{~T}>\mathrm{C}$ & p.F23S & 2 & NA & NA & ВП \\
\hline N2 & $\mathrm{O}$ & c.772G $>\mathrm{T}$ & p.G258C & 7 & NA & CM032578 & $\Pi$ \\
\hline N3 & $\mathrm{O}$ & c.926T $>C$ & p.L309P & 8 & NA & CM930307 & $\Pi$ \\
\hline N4 & 0 & c. $626 C>T$ & p.T209M & 6 & NA & CM940824 & $\Pi$ \\
\hline N5 & $\mathrm{H}$ & c. $455 \mathrm{~T}>\mathrm{C}$ & p.A152S & 4 & NA & NA & $\mathrm{H} \Pi$ \\
\hline N6 & 0 & c. $1019 \mathrm{G}>\mathrm{A}$ & p.S340N & 8 & NA & CM178801 & $\Pi$ \\
\hline N7 & $\mathrm{H}$ & c. $436 C>G$ & p.L146V & 4 & NA & NA & ВП \\
\hline N8 & $\mathrm{O}$ & $c .571 C>T$ & p.R191W & 5 & 0.000008 & CM001170 & $\Pi$ \\
\hline N9 & $\mathrm{O}$ & c. $974 \mathrm{G}>\mathrm{C}$ & p.R325P & 8 & NA & NA & $\mathrm{H} \Pi$ \\
\hline N10 & $\mathrm{O}$ & c. $666 C>\mathrm{G}$ & p.V222V & 6 & NA & NA & $\mathrm{H} \Pi$ \\
\hline N11 & $\mathrm{O}$ & c. $449 \mathrm{~T}>\mathrm{A}$ & p.F150Y & 4 & NA & CM097114 & $\Pi$ \\
\hline $\mathrm{N} 12$ & 0 & c. $478 \mathrm{G}>\mathrm{A}$ & p.D160N & 4 & NA & CM096832 & $\Pi$ \\
\hline
\end{tabular}

Примечания: наследственность (в 2 и более поколениях): О - отягощена; $\mathrm{H}$ - не отягощена или неизвестна. Патогенность: П - патогенный; ВП - возможно патогенный; НП - вариант с неопределенной патогенностью. gnomAD - browser beta (http://gnomad-beta.broadinstitute.org). HGMD - The Human Gene Mutation Database (http://www.hgmd.cf.ac.uk). NA - not available. Референсная последовательность (www.ncbi.nlm.nih.gov/nuccore): GCKNM_000162.3.

пациентов. Отсутствие антител к антигенам бета-клетки, особенно при наличии семейного характера заболевания, характерно для моногенных форм СД, но может наблюдаться менее чем у 5\% больных с СД 1 типа. Длительно сохраняющаяся эндогенная секреция инсулина (при длительности заболевания более 3-5 лет) не характерна для СД 1 типа и наблюдается при СД типа MODY. У всех пациентов обследуемой группы специфические панкреатические антитела были отрицательными.

Наличие в нескольких поколениях членов семьи с характерными нарушениями углеводного обмена позволяет с большой долей вероятности заподозрить моногенный характер заболевания, однако это также свойственно и СД 2 типа. Среди обследованных нами пациентов заболевание в большинстве случаев носило семейный характер: у десяти пациентов родственники первой степени родства имели нарушения углеводного обмена различной степени выраженности. В связи с бессимптомным течением заболевания нарушения углеводного обмена длительное время остаются недиагностированными, и только активное, целенаправленное обследование ближайших родственников позволяет выявить у них нарушения углеводного обмена. Так, у родственников шестерых пациентов нашей группы с нарушениями углеводного обмена были найдены аналогичные мутации методом прямого секвенирования по Сэнгеру.

Отсутствие признаков метаболического синдрома, ожирения является одним из характерных признаков СД типа MODY2 и, в ряде случаев, отличает его от СД 2 типа.

Нарушения углеводного обмена, свойственные СД типа MODY2, могут быть выявлены в любом возрасте. Умеренная непрогрессирующая гипергликемия, обусловленная мутациями в гене $G C K$, возникает с рождения, но, оставаясь бессимптомной длительное время, может быть впервые обнаружена после 18 лет. В сравнительном исследовании Т.Л. Кураевой и соавт. по изучению MODY2 и MODY3 у детей в России [2] было показано, что при
MODY2 гипергликемия определяется уже с рождения (в возрасте до 1 года у 13,4\%, минимальный возраст - 1 месяц), в отличие от MODY3 (минимальный возраст диагностики заболевания - 8 лет). Возможна ситуация, при которой молодые пациенты с неуточненным типом нарушения углеводного обмена или с нетипичным течением СД 1 типа переходят под наблюдение взрослых эндокринологов. В нашей группе у 7 из 12 пациентов нарушения углеводного обмена были выявлены в возрасте до 18 лет. Из них одной пациентке был поставлен диагноз СД 1 типа и назначена инсулинотерапия, другой - СД 2 типа, у троих тип СД не был определен и двое наблюдались с диагнозом НТГ.

Часто у пациентов с СД типа MODY2 результаты ПгTТ и значения $\mathrm{HbA}_{1 c}$ не соответствуют критериям СД, что вызывает трудности при формулировке диагноза. НТГ или нарушенная гликемия натощак не противоречат диагнозу СД типа MODY2, являясь его характерной особенностью. В разные периоды жизни пациента результаты ПГТТ и $\mathrm{HbA}_{1 с}$ могут незначительно меняться [25].

Во время беременности показатели гликемии у пациенток с СД типа MODY2 незначительно повышаются и, как правило, соответствуют критериям манифестного СД. Четырем пациенткам нашей группы во время беременности была назначена интенсифицированная инсулинотерапия с последующей отменой после родоразрешения (N4, N7, N11, N12). Одна пациентка (N6) продолжала соблюдать диету во время беременности. Так как СД типа MODY2 наследуется по аутосомно-доминантному типу, риск передачи данной мутации ребенку составляет 50\%. Выбор тактики лечения СД типа MODY2 во время беременности должен базироваться на генотипе плода: риску развития макросомии под влиянием материнской гипергликемии подвержены только младенцы, не имеющие мутации в гене GCK [26]. В случае же, когда мать и ребенок имеют мутации в гене $G C K$, гипергликемия не требует коррекции инсулином, а ребенок рождается с нормальным весом [16, 27]. Дети, которые наследуют мутацию, имеют тот же го- 
1. Возраст на момент постановки диагноза.

2. Пол.

3. В настоящее время лечение инсулином или ПССП?

4. Время до назначения инсулинотерапии (если в настоящее время лечится инсулином).

5. ИMT.

6. $\mathrm{HbA}_{1 \mathrm{c}}$.

7. Возраст на текущий момент.

8. У кого-то из родителей есть диабет?

лет

мужской

да

В настоящее время не лечится инсулином

В течение 6 месяцев после постановки диагноза

Более 6 месяцев после постановки диагноза

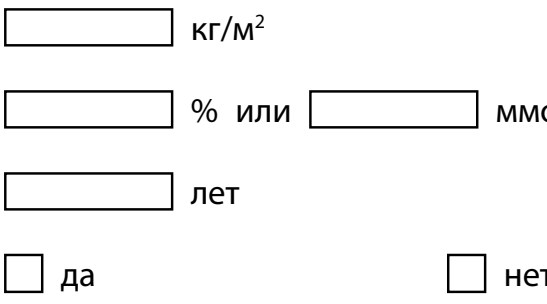

Рис. 2. Калькулятор MODY [5, 23]. Адаптировано из [30].

меостатический уровень глюкозы, что и их матери, и ощущают более высокий уровень глюкозы как нормальный. В этом случае гипергликемия не оказывает негативного влияния на вес ребенка. Риск развития осложнений при инвазивном методе пренатального скрининга с целью определения генотипа плода (если только амниоцентез/ хорионическая биопсия не осуществляется по более значимым причинам) является обоснованием для поиска путей неинвазивной пренатальной диагностики (в настоящее время у беременных могут проводиться УЗИ с допплерометрией, генетическое исследование родителей, кардиотокография, анализ крови на содержание сывороточных маркеров). Альтернативой пренатальному скринингу может быть ультразвуковая фетометрия каждые 2 нед, начиная с 26 нед беременности. При этом ускоренный фетальный рост косвенно указывает на отсутствие инактивирующей мутации в гене GCK у плода, что является показанием к инициации инсулинотерапии [28].

В 2012 г. A.T. Hattersley et al. [29] разработали простую модель прогнозирования MODY (используя логистическую регрессию), которая может помочь выявить случаи, подходящие для дальнейшего генетического тестирования. Модель улучшала чувствительность с 72\% до 91\% и специфичность от 91\% до 94\% для определения MODY по сравнению со стандартными критериями. Наиболее привлекательной является простота использования данной разработки (рис. 2). Для нашей пациентки Positive Predictive Value данного метода («положительное предсказательное значение», PPV) составляет 75,5\%, что позволяет предположить у нее MODY с высокой достоверностью. Однако данный онлайн-калькулятор может быть использован только у пациентов с манифестацией нарушений углеводного обмена в возрасте до 35 лет и, таким образом, формально не применим для пациентов с впервые диагностированными нарушениями углеводного обмена в возрасте от 35 лет и старше. К примеру, в нашей группе у мужчины с ожирением 2 степени впервые нарушения углеводного обмена диагностировали в возрасте 41 года (N10). Данный калькулятор дорабатывается и модифицируется [10, 29]. У беременных же используются иные критерии диагностики нарушений углеводного обмена, в связи с чем данный калькулятор имеет ограничения в этой группе пациентов.

Подтверждение инактивирующей мутации в гене GCK (MODY2), в отличие от других типов MODY, позволяет отказаться от малоэффективной в типичных случаях инсулинотерапии или пероральной сахароснижающей терапии. Так, в нашей группе 2 пациентам была отменена инсулинотерапия, 4 - пероральные сахароснижающие препараты. Все пациенты во время госпитализации достигли целевых уровней гликемии на фоне низкоуглеводной диеты.

\section{ЗАКЛЮЧЕНИЕ}

Такие клинические особенности заболевания, как пограничные длительно не прогрессирующие нарушения углеводного обмена (НГН, НТГ), отсутствие специфических аутоантител, отсутствие эффекта от проводимой пероральной сахароснижающей терапии у молодых пациентов с нормальной массой тела, особенно при наличии семейного характера заболевания, требуют проведения МГИ с целью исключения СД типа MODY2.

\section{ДОПОЛНИТЕЛЬНАЯ ИНФОРМАЦИЯ}

Согласие пациентов. При поступлении в стационар пациентка дала согласие на обработку персональных данных. Информированное согласие на публикацию медицинских данных, представленных в статье (в обезличенной форме) в журнале «Сахарный диабет», от пациентки получено.

Конфликт интересов. Авторы декларируют отсутствие явных и потенциальных конфликтов интересов, связанных с публикацией настоящей статьи.

Участие авторов. Кононенко И.В. - концепция и дизайн исследования, набор материала, редактирование текста; Глибка А.А. - обработка материала, обзор литературы, написание текста, оформление рисунков; Зубкова Н.А. - анализ результатов МГИ, редактирование текста; Майоров А.Ю. - окончательное утверждение рукописи для публикации; Тюльпаков А.Н. - проведение МГИ, окончательное утверждение рукописи для публикации; Шмидт О.М. - набор материала. Все авторы внесли существенный вклад в проведение исследования и подготовку статьи, прочли и одобрили финальную версию перед публикацией. 


\section{СПИСОК ЛИТЕРАТУРЫ | REFERENCES}

1. Shields BM, Peters JL, Cooper C, et al. Can clinical features be used to differentiate type 1 from type 2 diabetes? A systematic review of the literature. BMJ Open. 2015;5(11):e009088. doi: https://doi.org/10.1136/bmjopen-2015-009088

2. Кураева ТЛ., Сечко Е.А., Зильберман Л.И., и др. Молекулярногенетические и клинические варианты MODY2 и MODY3 у детей в России // Проблемы эндокринологии. — 2015. — Т. 61. — №5. C. 14-25. [Kuraeva TL, Sechko EA, Zilberman LI, et al. Molecular genetic and clinical variants MODY2 and MODY3 in children in Russia. Problems of Endocrinology. 2016;61 (5):14-25. (In Russ.)] doi: https://doi.org/10.14341/probl201561514-25

3. Pihoker C, Gilliam LK, Ellard S, et al. Prevalence, Characteristics and Clinical Diagnosis of Maturity Onset Diabetes of the Young Due to Mutations in HNF1A, HNF4A, and Glucokinase: Results From the SEARCH for Diabetes in Youth. J Clin Endocrinol Metab. 2013;98(10):4055-4062. doi: https://doi.org/10.1210/jc.2013-1279

4. Bishay RH, Greenfield JR. A review of maturity onset diabetes of the young (MODY) and challenges in the management of glucokinase-MODY. Med J Aust. 2016;205(10):480-485. doi: https://doi.org/10.5694/mja16.00458

5. Amed S, Dean HJ, Panagiotopoulos C, et al. Type 2 Diabetes, Medication-Induced Diabetes, and Monogenic Diabetes in Canadian Children: A prospective national surveillance study. Diabetes Care. 2010;33(4):786-791. doi: https://doi.org/10.2337/dc09-1013

6. DellaManna T, Silva MRd, Chacra AR, et al. Clinical follow-up of two Brazilian subjects with glucokinaseMODY (MODY2) with description of a novel mutation. Ara Bras Endocrinol Metabol. 2012;56(8):490-495. doi: https://doi.org/10.1590/s0004-27302012000800005

7. Pruhova S, Dusatkova P, Sumnik Z, et al. Glucokinase diabetes in 103 families from a country-based study in the Czech Republic: geographically restricted distribution of two prevalent GCK mutations. Pediatr Diabetes. 2010;11(8):529-535. doi: https://doi.org/10.1111/j.1399-5448.2010.00646.x

8. Stern E, Strihan C, Potievsky O, et al. Four novel mutations, including the first gross deletion in TCF1, identified in HNF-4alpha, GCK and TCF1 in patients with MODY in Israel. J Pediatr Endocrinol Metab. 2007;20(8):909-921. doi: https://doi.org/10.1515/jpem.2007.20.8.909

9. Shields BM, Hicks S, Shepherd MH, et al. Maturityonset diabetes of the young (MODY): how many cases are we missing? Diabetologia. 2010;53(12):2504-2508 doi: https://doi.org/10.1007/s00125-010-1799-4

10. Firdous P, Nissar K, Ali S, et al. Genetic Testing of MaturityOnset Diabetes of the Young Current Status and Future Perspectives. Front Endocrinol (Lausanne). 2018;9. doi: https://doi.org/10.3389/fendo.2018.00253

11. Chakera AJ, Steele AM, Gloyn AL, et al. Recognition and Management of Individuals With Hyperglycemia Because of a Heterozygous Glucokinase Mutation. Diabetes Care. 2015;38(7):1383-1392. doi: https://doi.org/10.2337/dc14-2769

12. Njolstad PR, Sagen JV, Bjorkhaug L, et al. Permanent Neonatal Diabetes Caused by Glucokinase Deficiency: Inborn Error of the Glucose-Insulin Signaling Pathway. Diabetes. 2003;52(11):28542860. doi: https://doi.org/10.2337/diabetes.52.11.2854

13. Singh R, Pearson ER, Clark PM, Hattersley AT. The long-term impact on offspring of exposure to hyperglycaemia in utero due to maternal glucokinase gene mutations. Diabetologia. 2007:50(3):620-624. doi: https://doi.org/10.1007/s00125-006-0541-8

14. Зубкова Н.А., Бурумкулова Ф.Ф., Улятовская В.И., и др. Весо-ростовые показатели детей, рожденных от матерей с гестационным сахарным диабетом, обусловленным мутациями в гене глюкокиназы // Сахарный диабет. - 2018 - Т. 21. — №2. C. 92-98. [Zubkova NA, Burumkulova FF, Ulyatovskaya VI, et al.
Birth weight and length in offsprings of mothers with gestational diabetes mellitus due to mutations in GCK gene. Diabetes mellitus. 2018;21 (2):92-98. (In Russ.)] doi: https://doi.org/10.14341/DM9429

15. Меликян М.А., Карева М.А. Врожденный гиперинсулинизм: Пособие для врачей. - M.; 2017. [Melikyan MA, Kareva MA. Vrozhdennyy giperinsulinizm: Manual for doctors. Moscow; 2017. (In Russ.)]

16. Anık A, C atlı G, Abacı A, Böber E. Maturity-onset diabetes of the young (MODY): an update. J Pediatr Endocrinol Metab. 2015;28(3-4). doi: https://doi.org/10.1515/jpem-2014-0384

17. Ellard S, Beards F, Allen LIS, et al. A high prevalence of glucokinase mutations in gestational diabetic subjects selected by clinical criteria. Diabetologia. 2000;43(2):250-253. doi: https://doi.org/10.1007/s001250050038

18. Lachance C-H. Practical Aspects of Monogenic Diabetes: A Clinical Point of View. Can J Diabetes. 2016;40(5):368-375. doi: https://doi.org/10.1016/j.jcjd.2015.11.004

19. Кураева ТЛ., Зильберман Л.И., Титович Е.В., и др. Генетика моногенных форм сахарного диабета // Сахарный диабет. 2011 - T. 14. — №1. - C. 20-27. [Kuraeva TL, Zil'berman LI, Titovich EV, et al. Genetics of monegenic forms of diabetes mellitus. Diabetes mellitus. 2011;14(1):20-27. (In Russ.)] doi: https://doi.org/10.14341/2072-0351-6246.

20. Froguel $P$, Vaxillaire $M$, Sun $F$, et al. Close linkage of glucokinase locus on chromosome 7p to early-onset non-insulindependent diabetes mellitus. Nature. 1992;356(6365):162-164. doi: https://doi.org/10.1038/356162a0

21. Солвей Дж.Г. Наглядная медичинская биохимия:учебное пособие. / Под ред. Северина Е.С. - М.: ГЭОТАР-Медиа; 2015. [Solvay JG. Severin ES, editor. Naglyadnaya meditsinskaya biokhimiya: tutorial. Moscow: GEOTAR-Media; 2015. (In Russ.)]

22. Lu B, Munoz-Gomez M, Ikeda Y. The two major glucokinase isoforms show conserved functionality in $\beta$-cells despite different subcellular distribution. Biol Chem. 2018;399(6):565-576. doi: https://doi.org/10.1515/hsz-2018-0109

23. hgmd.cf.ac.uk [Internet]. The Human Gene Mutation Database [cited 2019 Jan 23]. Available from: http://www.hgmd.cf.ac.uk/ac/index.php.

24. Osbak KK, Colclough K, Saint-Martin C, et al. Update on mutations in glucokinase (GCK), which cause maturity-onset diabetes of the young, permanent neonatal diabetes, and hyperinsulinemic hypoglycemia. Hum Mutat. 2009;30(11):15121526. doi: https://doi.org/10.1002/humu.21110

25. Froguel $\mathrm{P}$, Zouali $\mathrm{H}$, Vionnet $\mathrm{N}$, et al. Familial Hyperglycemia Due to Mutations in Glucokinase -- Definition of a Subtype of Diabetes Mellitus. New Eng J Med. 1993;328(10):697-702. doi: https://doi.org/10.1056/nejm199303113281005

26. Velho G, Hattersley AT, Froguel P. Maternal diabetes alters birth weight in glucokinase-deficient (MODY2) kindred but has no influence on adult weight, height, insulin secretion or insulin sensitivity. Diabetologia. 2000;43(8):1060-1063. doi: https://doi.org/10.1007/s001250051490

27. Colom C, Corcoy R. Maturity onset diabetes of the young and pregnancy. Best Pract Res Clin Endocrinol Metab. 2010;24(4):605-615. doi: https://doi.org/10.1016/j.beem.2010.05.008

28. Dickens LT, Naylor RN. Clinical Management of Women with Monogenic Diabetes During Pregnancy. Curr Diab Rep. 2018;18(3). doi: https://doi.org/10.1007/s11892-018-0982-8

29. Niølstad PR, Molven A. To test, or not to test: time for a MODY calculator? Diabetologia. 2012;55(5):1231-1234. doi: https://doi.org/10.1007/s00125-012-2514-4

30. diabetesgenes.org [Internet]. MODY Probability Calculator [cited 2019 Jan 23]. Available from: https://www.diabetesgenes.org/mody-probability-calculator . 
ИНФОРМАЦИЯ ОБ АВТОРАХ [AUTHORS INFO]

*Глибка Анастасия Андреевна, аспирант [Anastasiya A. Glibka, MD, PhD student]; адрес: Россия, 117036, Москва, улица Дм. Ульянова, д. 11 [address: 11 Dm.Ulyanova street, 117036 Moscow, Russian Federation]; ORCID: https://orcid.org/0000-0002-7324-8344; eLibrary: SPIN 3649-6340; e-mail: anastasiya_glibka@mail.ru

Кононенко Ирина Владимировна, к.м.н., доцент [Irina V. Kononenko, MD, PhD, associate professor]; ORCID: https://orcid.org/0000-0003-4929-1526; eLibrary: SPIN 6528-7986; e-mail: shakhtarina@bk.ru Майоров Александр Юрьевич, д.м.н., профессор [Alexander Y. Mayorov, MD, PhD, Professor]; ORCID: https://orcid.org/0000-0001-5825-3287; eLibrary SPIN: 4275-7779; e-mail: education@endocrincentr.ru. Зубкова Наталья Анатольевна, к.м.Н., С.н.c. [Natalya A. Zubkova, MD, PhD, senior research associate]; ORCID: https://orcid.org/0000-0002-1346-7545; eLibrary: SPIN: 5064-9992; e-mail: zunata2006@yandex.ru Тюльпаков Анатолий Николаевич, д.м.н., професcop [Anatoly N. Tyulpakov, MD, PhD, Professor]; ORCID: https://orcid.org/0000-0001-8500-4841; eLibrary SPIN: 8396-1798; e-mail: ant@endocrincentr.ru Шмидт Ольга Марковна, врач-эндокринолог [Olga M. Schmidt, MD]; ORCID: https://orcid.org/0000-0002-5033-2412; eLibrary SPIN: 6118-3196; e-mail: olga_shm@rambler.ru

\section{ЦИТИРОВАТЬ:}

Кононенко И.В., Глибка А.А., Зубкова Н.А., Майоров А.Ю., Тюльпаков А.Н., Шмидт О.М. Трудности диагностики сахарного диабета типа MODY2 у взрослых // Сахарный диабет. - 2019. — T. 22. — №4. — C. 384-391. doi: 10.14341/DM10063

\section{TO CITE THIS ARTICLE:}

Kononenko IV, Glibka AA, Zubkova NA, Mayorov AY, Tyulpakov AN, Schmidt OM. MODY2 diagnostic issues in adults. Diabetes Mellitus. 2019;22(4):384-391. doi: 10.14341/DM10063 